\title{
Evaluation of chitosan based vaginal bioadhesive gel formulations for antifungal drugs
}

\author{
ZEYNEP AY ŞENYIĞGiT \\ SINEM YAPRAK KARAVANA ${ }^{1}$ \\ BAYRI ERAÇ² \\ ÖZGE GÜRSEL ${ }^{1}$ \\ MINE HOŞGÖR LIMONCU² \\ ESRA BALOĞLU ${ }^{1}$ \\ ${ }^{1}$ Ege University, Faculty of Pharmacy \\ Department of Pharmaceutical Technology \\ 35100 Bornova, İzmir, Turkey \\ ${ }^{2}$ Ege University, Faculty of Pharmacy \\ Department of Microbiology \\ 35100 Bornova, İmir, Turkey
}

\begin{abstract}
The aim of the present study was to evaluate chitosan as a vaginal mucoadhesive gel base for econazole nitrate and miconazole nitrate. To this aim, different types of chitosan with different molecular masses and viscosity properties [low molecular mass chitosan (viscosity: 20,000 mPa s), medium molecular mass chitosan (viscosity: 200,000 $\mathrm{mPa}$ s), high molecular mass chitosan (viscosity: $800,000 \mathrm{mPa} \mathrm{s}$ )] have been used. First, rheological studies were conducted on chitosan gels. Mechanical, syringeability and mucoadhesive properties of chitosan gels were determined. Release profiles of econazole nitrate and miconazole nitrate from chitosan gels were obtained and evaluated kinetically. In addition, anticandidal activities of formulations were determined. Finally, vaginal retention of chitosan gels in rats was evaluated by in vivo distribution studies. Based on the results, it can be concluded that gels prepared with medium molecular mass chitosan might be effectively used for different antifungal agents in the treatment of vaginal candidiosis, since it has high mucoadhesiveness, suitable mechanical and release properties with good vaginal retention.
\end{abstract}

Keywords: chitosan, vaginal gel, mucoadhesion, econazole nitrate, miconazole nitrate, vaginal retention

Vaginal yeast infections are among the most common reasons for women seeking medical care; it has been estimated that $70-75 \%$ of women have an episode of Candida vaginitis at least once during their lifetime (1). Local administration of antimicrobial agents has been favored in the treatment of vaginitis because of the numerous side effects of systemically applied drugs. Vaginal dosage forms used for this purpose include solutions, gels, creams, ointments, foams, pessaries, tablets and vaginal inserts (1). To achieve a desirable therapeutic effect, vaginal delivery systems need to have the ability to spread onto the vaginal mucosa surface and guarantee an intimate and prolonged contact at the site of application (2). Therefore, localized mucoadhesive dosage forms

\footnotetext{
* Correspondence; e-mail: zeynepay79@hotmail.com
} 
may be regarded as a suitable formulation design to improve both the bioavailability of the drug and patient compliance while traditional vaginal preparations might not ensure satisfactory therapy.

Mucoadhesive gels have a high capability of adhering to mucosal surfaces, which cover various body cavities such as the vagina and prolong the residence time of the drug delivery system at the application site and decrease the number of applications required (3). Use of natural polymers is valuable for the preparation of mucoadhesive gel formulations owing to their proven biocompatibility and safety. In this respect, the polysaccharide chitosan has received particular attention over the past 20 years (4). Chitosan is a natural copolymer consisting of glucosamine and $\mathrm{N}$-acetylglucosamine units. It has a cationic character based on its primary amino groups. These primary amino groups of chitosan are responsible for various properties (i.e., controlled drug release, mucoadhesion, in situ gelation and permeation enhancement). In addition, chitosan and its derivatives have efflux pump inhibitory properties. They are able to reversibly inhibit efflux pumps and owing to this property the mucosal uptake of various efflux pump substrates (such as anticancer drugs, antimycotic drugs and antiinflammatory drugs) can be greatly improved (4). All of these properties make chitosan an ideal candidate for being used in drug delivery systems $(4,5)$.

Miconazole nitrate (MN) and econazole nitrate (EN) are imidazole derivative antifungal drugs used to treat topical fungal and yeast infections (6). The objective of this study was to comprehensively evaluate chitosan as a vaginal gel base for EN and MN. For this purpose, different types of chitosan with different molecular masses and viscosity properties have been used for the preparation of mucoadhesive gels. First of all, a comprehensive rheological study was conducted on prepared chitosan gels. Mechanical, syringeability and mucoadhesion properties of chitosan gels were determined with a TA-XT Texture Analyzer (7). Release profiles of MN and EN were obtained and evaluated kinetically. In addition, anticandidal activities of formulations were determined by the agar well diffusion method. Finally, the vaginal retention of chitosan gels in rats was evaluated by in vivo distribution studies.

\section{EXPERIMENTAL}

\section{Materials}

Low molecular mass chitosan [Brookfield viscosity $(1 \%, \mathrm{~m} / \mathrm{V}$, in $1 \%$ acetic acid solution at $\left.25{ }^{\circ} \mathrm{C}\right): 20,000 \mathrm{mPa} \mathrm{s}, 75-85 \%$ deacetylated], medium molecular mass chitosan [Brookfield viscosity $\left(1 \%, m / V\right.$, in $1 \%$ acetic acid solution at $\left.25^{\circ} \mathrm{C}\right): 200,000 \mathrm{mPa} s, 75-85$ $\%$ deacetylated], high molecular mass chitosan [Brookfield viscosity $(1 \%, m / V$, in $1 \%$ acetic acid solution at $25{ }^{\circ} \mathrm{C}$ ): $800,000 \mathrm{mPa} s,>75 \%$ deacetylated] and EN were purchased from Sigma-Aldrich (GERMANY). MN was obtained from İLSAN-İLTAŞ Pharmaceutical Company (TURKEY). All other materials were of analytical grade. 


\section{Preparation of formulations}

Gel formulations were prepared using chitosan of three different molecular masses. Briefly, chitosan of $2 \%(\mathrm{~m} / \mathrm{m})$ concentration was dissolved in diluted lactic acid solution $(1 \%, V / V)$. Percent chitosan concentration was chosen according to literature findings and preformulation studies (8). Chitosan concentration in gel formulations was kept constant to evaluate the difference between chitosan types. MN or EN was incorporated into formulations at $2 \%(\mathrm{~m} / \mathrm{m})$ and $1 \%(\mathrm{~m} / \mathrm{m})$ concentrations, respectively. MN and EN concentrations were chosen in accordance with their concentration in commercial products. Compositions of gels are given below:

F1: Formulations prepared with low molecular mass chitosan (2\%)

F2: Formulations prepared with medium molecular mass chitosan (2\%)

F3: Formulations prepared with high molecular mass chitosan $(2 \%)$.

\section{Determination of $p H$}

The $\mathrm{pH}$ values of gels were measured with a $\mathrm{pH}$-meter (WTW series $\mathrm{pH} 720$ ) at room temperature. The $\mathrm{pH}$ values of formulations were checked 24 hours upon preparation.

\section{Rheological studies}

Rheological analysis of chitosan gels was performed at $37 \pm 0.1{ }^{\circ} \mathrm{C}$ with a Haake Mars rheometer (Thermo Fisher, Germany). Analysis was performed in flow mode and in conjunction with parallel steel plate geometry (40 mm diameter) with a gap of 0.3 $\mathrm{mm}$, as described previously (9). In continuous shear analysis, the upward and downward flow curves for each formulation were measured over shear rates (10-2000 s $\left.\mathrm{s}^{-1}\right)$.

Oscillatory analysis of each formulation was performed after determination of its linear viscoelastic region at $37 \pm 0.1^{\circ} \mathrm{C}$, where stress was directly proportional to strain and the storage modulus remained constant. Frequency sweep analysis was performed over the frequency range of $0.1-10.0 \mathrm{~Hz}$ following application of a constant stress. The standard gap size was $0.3 \mathrm{~mm}$ for each sample. Storage modulus $\left(G^{\prime}\right)$ and loss modulus $\left(G^{\prime \prime}\right)$ of the formulations were determined as previously described (10). In each case, rheological properties of the formulations were examined at least six times.

\section{Mechanical properties of formulations}

Textural analysis was performed to determine mechanical properties of the formulations using a software-controlled penetrometer (TA-TX Plus, Stable Micro System, UK) equipped with a $5 \mathrm{~kg}$ load cell. Formulations were initially transferred into glass vials (20 $\mathrm{mL})$. An analytical probe was twice compressed into each formulation to a defined depth $(15 \mathrm{~mm})$ and at a defined rate $\left(2 \mathrm{~mm} \mathrm{~s}^{-1}\right)$, allowing a delay period (15 s) between the end of the first and beginning of the second compression. Experiments were carried out at least six times. As a result of this study, a force-time curve was obtained (Fig. 1) and mechanical parameters (hardness, compressibility, adhesiveness, cohesiveness and elasticity) were calculated (11). 
Hardness is the force required to attain a given deformation and the altitude of the first peak gives the hardness value. Compressibility is the work required to deform the product during the first compression of the probe and the first area under the curve $(A U C)$ defines compressibility. Adhesiveness is the work necessary to overcome the attractive forces between the surface of the sample and the surface of the probe. The second AUC defines adhesiveness. Cohesiveness is the ratio of the area under the force-time curve produced in the second compression cycle to that in the first compression cycle. Elasticity is the rate at which the deformed sample returns to its undeformed condition after the removal of the deforming force. Cohesiveness and elasticity values were calculated according to the following equations:

$$
\text { Cohesiveness }=A U C_{3} / A U C_{1}
$$

$$
\text { Elasticity }=\text { Time }^{\text {difference }}{ }_{3-4} / \text { Time difference }_{1-2}
$$

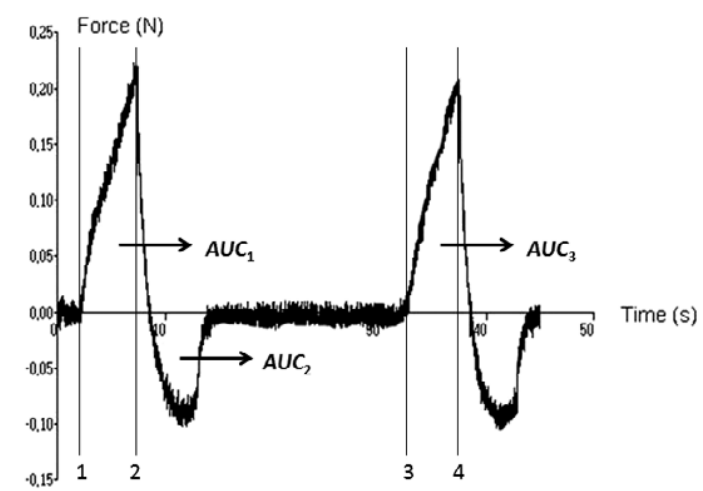

Fig. 1. Schematic representation of the force-time curve obtained from textural analysis.

\section{Syringeability of formulations}

The work required to expel formulations from a syringe without a needle was measured using a TA-XT Texture Analyzer (Stable Micro Systems) in compression mode, as previously described in literature (12). Shortly, formulations were packed into $2 \mathrm{~mL}$ plastic syringes without needles to a height of $25 \mathrm{~mm}$, avoiding air bubbles. The syringe was then placed in the metallic support and immobilized with a plastic clamping ring. A force transducer was connected to the texture analyzer TA-XT to measure the force with which the arm of the texture analyzer moved and pushed the plunger of the syringe. The probe was lowered at a constant speed $\left(2.0 \mathrm{~mm} \mathrm{~s}^{-1}\right)$ over a distance of $30 \mathrm{~mm}$. The resistance to expression of syringe contents (work done) was determined from the area under the force-time curve recorded during plunger compression. All measurements were performed in at least six replicates (12). 


\section{Mucoadhesion studies}

Mucoadhesion measurements were performed using a TA-XT Texture Analyzer (Stable Micro Systems) equipped with a load cell of $1 \mathrm{~kg}$. A/MUC measuring system (mucoadhesion test ring) consisting of a ring in which the biological support can be fixed and a cylinder probe with a diameter of $1 \mathrm{~cm}$ were used. In this study, mucin dispersion (mucin from porcine stomach, Type II, Sigma-Aldrich, Germany) was used instead of a biological support. A filter paper disc was wetted with $10 \mu \mathrm{L}$ of mucin dispersion in phosphate buffer $(8 \%, \mathrm{~m} / \mathrm{m}, \mathrm{pH} 6.4) .20 \mathrm{mg}$ of each sample was applied to the cylinder probe. The sample and biologic substrate were put in contact with a preload of $6000 \mathrm{mN}$ for $3 \mathrm{~min}$. The cylinder probe was moved upwards at a predetermined speed of $2.5 \mathrm{~mm} \mathrm{~min}^{-1}$ to separate the mucoadhesive interface (mucin-sample). The maximum detachment force, as a function of displacement, was recorded and the work of adhesion parameter $(\mathrm{mN} \mathrm{mm})$ was calculated as the area under the force $v$ s. displacement curve. The test was conducted at $37^{\circ} \mathrm{C}$ and each experiment was carried out six times (13).

\section{Release studies}

The in vitro release studies of $\mathrm{MN}$ and $\mathrm{EN}$ from different chitosan gel bases were carried out using Spectra/Por Regenerated Cellulose Dialysis Membrane Tubes (12.000-14.000 MWCO). Membranes were soaked in distilled water for 24 hours before the experiment. Phosphate buffer ( $\mathrm{pH} 4.5)$ with dioxane $(65: 35, V / V)$ was used as receptor medium (14). Each formulation was packed in dialysis bags, which were placed in $150 \mathrm{~mL}$ of buffer at $37^{\circ} \mathrm{C}$ in sealed glass vials. The receptor medium was continuously stirred. At certain time intervals, samples were withdrawn and the drug content of each sample was analyzed spectrophotometrically (Shimadzu UV-Vis 1208 spectrophotometer). Samples were measured at $273 \mathrm{~nm}$ and $270 \mathrm{~nm}$ for MN and EN contents, respectively. All the experiments were repeated at least three times.

\section{Determination of release mechanism}

The release mechanism from gels was investigated using the Ritger-Peppas equation $(15,16)$.

$$
M_{\mathrm{t}} / M_{\infty}=k t^{n}
$$

In this equation; $M_{\mathrm{t}}$ is the drug released at time $t, M_{\infty}$ is the quantity of drug released at infinite time, $M_{t} / M_{\infty}$ is fractional release, $k$ is the diffusional constant and $n$ is the diffusional exponent that characterizes the drug release mechanism.

\section{Determination of anticandidal activities of formulations}

Anticandidal activities of formulations were determined by the agar well diffusion method. The standard Candida albicans ATCC 90028 strain was inoculated onto a Sabouraud Dextrose Agar (SDA, Oxoid) plate and incubated at $35{ }^{\circ} \mathrm{C}$ for 48 hours. A suspension equal to the $0.5 \mathrm{McF}$ arland turbidity standard was prepared in a sterile sa- 
line solution $\left(8.5 \mathrm{~g} \mathrm{~L}^{-1}\right)$ from the candida colonies. $100 \mu \mathrm{L}$ of the suspension containing approximately $1 \times 10^{6}-5 \times 10^{6}$ colony forming units (CFU)/mL was spread on the plates with a sterile swab and allowed to dry at room temperature for 5 minutes. Then, $20 \mathrm{~mm}$ diameter wells were made in the centers of SDA plates with a sterile scalpel. 0.5 $\mathrm{mL}$ of formulation with or without $\mathrm{MN}$ and $\mathrm{EN}$ was put into the wells aseptically. After incubation for $24-48$ hours at $35^{\circ} \mathrm{C}$, inhibition zone diameters formed around the formulations were measured. To control the possible contamination of formulations, they were placed on SDA plates that were not inoculated with C. albicans and incubated (17).

\section{In vivo distribution studies}

For in vivo distribution studies, the near-infrared dye IRDye 800RS Carboxylate (LI-COR Biosciences, NE) was loaded in chitosan gels $(10 \mathrm{mg} / 10 \mathrm{~g}, \mathrm{~m} / \mathrm{m})$. Female Wistar albino rats (200-250 g) were anesthetized with ketamine. Vaginal and abdominal regions of the rats were shaved and $0.2 \mathrm{~mL}$ of gels was given intravaginally using a syringe without a needle. Rats were transferred to the MousePOD ${ }^{\mathrm{TM}}$ Adapter (model 9201-MP) scanning surface of an Odyssey Infrared Imaging System (model 9201-3; LI-COR Biosciences, USA). The MousePOD ${ }^{\mathrm{TM}}$ adapter was equipped with temperature regulators to maintain animal temperature during scanning. Vaginal regions of animals were scanned from tail to abdomen in two infrared channels simultaneously (700 and $800 \mathrm{~nm}$ ), where one channel (700 nm channel) was used for normalization of the measured infrared intensities. The rats were scanned every hour for $8 \mathrm{~h}$ and $24 \mathrm{~h}$ after administration. The intensities were displayed in pseudo colors by the Odyssey software (LI-COR Biosciences) to isolate regions of interest (18). This in vivo study was performed following institutional approval from the Ethics Committee of Adnan Menderes University (Aydın, Turkey, No: B.30.2.ADU.0.00.00.00/050.04/2012/107). Experiments were carried out in accordance with the guidelines laid down by the Helsinki Declaration regarding the care and use of animals for experimental procedures and in accordance with local laws and regulations.

\section{Statistical data analysis}

Statistical data analysis was performed using the Student $t$-test with $p<0.05$ as the minimal level of significance.

\section{RESULTS AND DISCUSSION}

\section{Results of $\mathrm{pH}$ measurements}

The vaginal $\mathrm{pH}$ of healthy women of reproductive age is acidic (4.5-5.5). It is maintained by Lactobacilli present in the healthy vagina that play an important role in the control of infection by common pathogens. These organisms also produce other bactericidal compounds such as hydrogen peroxide, bacteriocin-like substances and possibly biosurfactants (19). The $\mathrm{pH}$ of formulations is one of important parameters for vaginal compatibility. Therefore, the $\mathrm{pH}$ values of chitosan gels were determined first in this 
Table I. $p H$ values of formulations

\begin{tabular}{cc}
\hline Formulation code & $\mathrm{pH} \pm \mathrm{SD}$ \\
\hline $\mathrm{F} 1$ & $5.34 \pm 0.04$ \\
$\mathrm{~F} 2$ & $5.44 \pm 0.06$ \\
$\mathrm{~F} 3$ & $4.98 \pm 0.03$ \\
$\mathrm{~F} 1+\mathrm{MN}$ & $4.82 \pm 0.07$ \\
$\mathrm{~F} 2+\mathrm{MN}$ & $4.67 \pm 0.08$ \\
$\mathrm{~F} 3+\mathrm{MN}$ & $4.53 \pm 0.04$ \\
F1 + EN & $3.85 \pm 0.06$ \\
F2 + EN & $3.92 \pm 0.09$ \\
F3 + EN & $3.79 \pm 0.07$ \\
\hline
\end{tabular}

study. The results showed that all the formulations were suitable for vaginal application as they could maintain the acidic $\mathrm{pH}$ value of the vagina (Table I). It was also observed that the $\mathrm{pH}$ value of the gels did not change significantly when using different types of chitosan. Addition of $\mathrm{EN}$ and $\mathrm{MN}$ into gel formulations significantly decreased $\mathrm{pH}$ values in all formulations $(p<0.05)$.

\section{Results of rheological studies}

Rheological properties affect the in vivo behavior of formulations in the vagina such as application, spreadability and retention time. Spreadability of gels increased with a decrease in viscosity. Also, the retention time of chitosan gels was affected by their viscosity and rheological behavior. Therefore, were evaluated the rheological behavior of chitosan gels and determined if the flow types of the formulations were Newtonian or non-Newtonian.

It can be seen from Fig. 2 that the gels exhibited flow behavior in dependence on the chitosan type. F1 formulation showed Newtonian flow due to its low viscosity. This flow behavior is common in diluted gel and emulsion formulations. The reason for Newtonian flow behavior is that the molecules or micelles of this formulation did not get entangled. Further, the formulation did not undergo a phase change to turn into a gel and was still an easily flowing liquid, similar to pure water displaying typical Newtonian flow behavior $(20,21)$. Solutions began to flow after shear stress reached its yield point. Therefore, the flow curve for these solutions, under physiological conditions, exhibited pseudoplastic behavior with hysteresis. The hysteresis phenomenon indicated the presence of an elastic component. Thus, strong gels formed with these polymers can be characterized as viscoelastic materials. Viscoelastic materials normally show hysteresis under cyclic deformation. Viscoelastic materials are preferable to viscous materials because they flow more easily and are less susceptible to deformation due to stress (21). On the other hand, F2 and F3 formulations exhibited plastic flow. Plastics are shear thinning, their curve begins with a yield value and their viscosity decreases with an increase in shear rate $(20,21)$. It is clear that the type of chitosan affects the flow behavior of a formulation. 

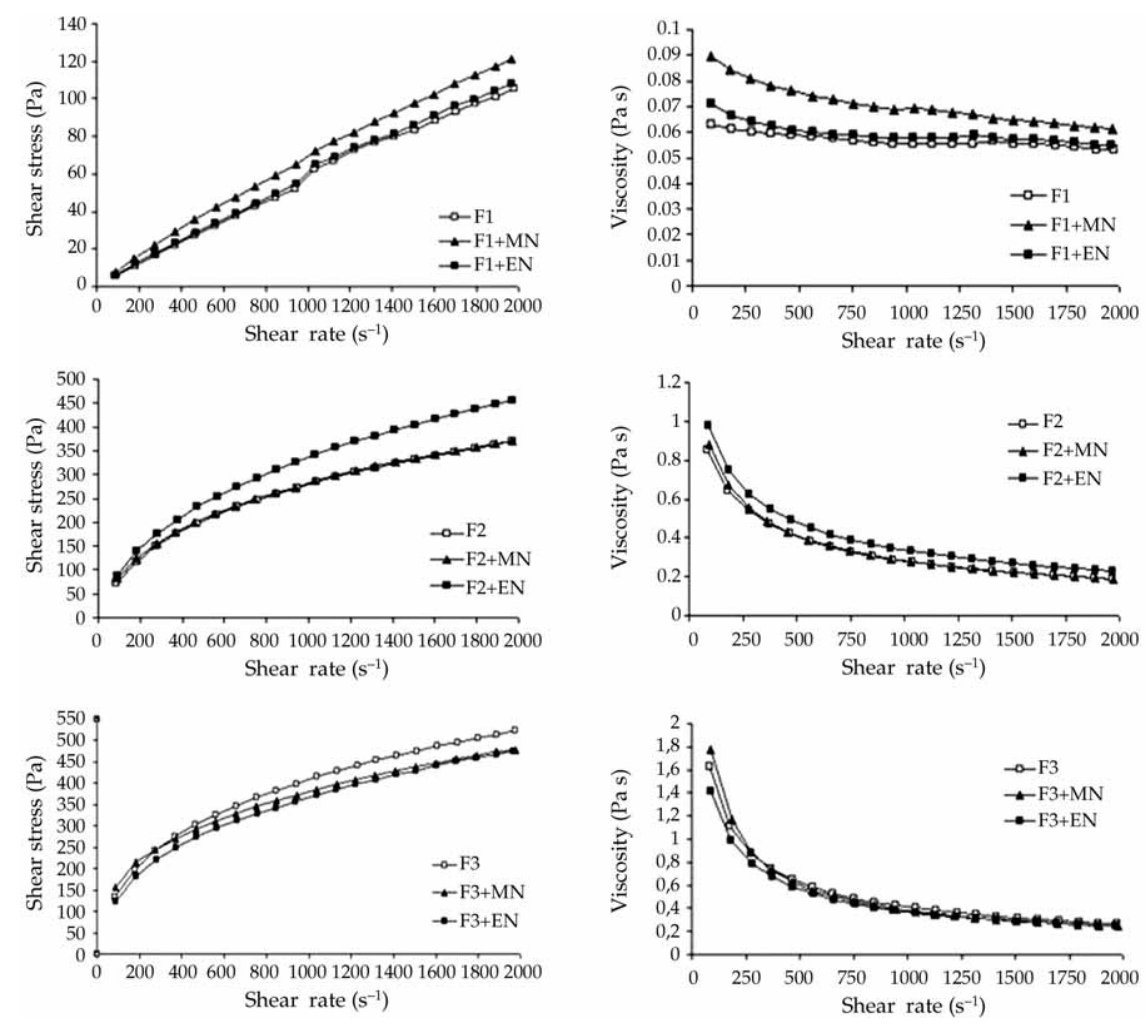

Fig. 2. Flow curves of chitosan gels.

Oscillatory studies determine the viscoelastic property of the formulation by subjecting the sample to sinusoidal shear stress. The effects of chitosan type and addition of an active substance on the viscoelastic properties, namely, storage modulus ( $G$ ', a measure of elasticity) and loss modulus ( $G^{\prime \prime}$, viscous components) of the formulations, are displayed in Fig. 3. As can be seen, these chitosan gels are characterized by lower $G^{\prime}$ and higher $G^{\prime \prime}$ values, pointing to a mainly viscous polymer solution containing a network established by physical entanglements. Also, at all frequency values a liquid-like behavior is observed, so the viscous properties are more pronounced than the elastic ones (22). Similar liquid type behavior has been reported for chitosan gels in the literature (22-24).

Results of our rheological studies also showed that the addition of MN or EN had no significant effect on the flow behavior or viscosity values for all formulations $(p>0.05)$.

\section{Mechanical properties of formulations}

Vaginal semi-solid formulations should have appropriate mechanical properties (e.g., mucoadhesive properties, ease of application, low hardness, and good retention at the application site) to provide maximum benefit of the formulation to the patient (12). 

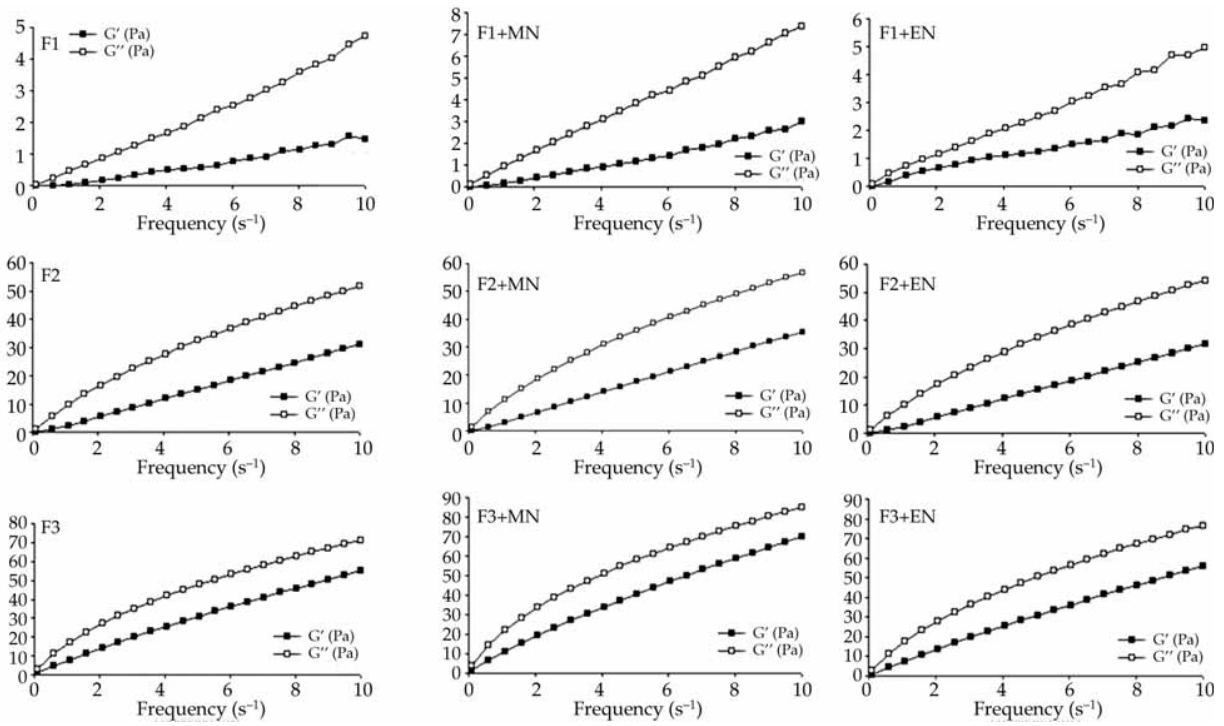

Fig. 3. Effects of chitosan type and addition of an active substance on storage modulus (G') and loss modulus $\left(\mathrm{G}^{\prime \prime}\right)$.

Therefore, vaginal bioadhesive gel formulations were evaluated according to their mechanical properties and the results are given in Table II.

Adhesiveness is the work required to detach the probe from the sample and is related to bioadhesion. Cohesiveness provides information on the effects of repeated shearing stresses on the structural properties of formulations. Hardness expresses the applicability of the gel to the desired site. Compressibility determines the extraction of the gel from the container and the simplicity of its spreadability on the application site. Elasticity represents the rate at which the deformed sample returns to its undeformed condition. Lower numerical values as determined by the Texture Profile Analysis (TPA) in elasticity mode indicate higher product elasticity (11). Formulations should have good adhesiveness properties to remain on the vaginal mucosa for a long period of time and thereby decrease application frequency. On the other hand, compressibility and hardness values of the formulations should be low to facilitate product removal from the container and provide product comfort within the vaginal cavity.

As can be seen from Table II, increase in chitosan molecular mass and viscosity altered the mechanical properties of all formulations. Formulations prepared with high molecular mass hitosan exhibited the highest adhesiveness values. On the other hand, the hardness and compressibility values of these formulations were, as expected, higher than the others, which might be disadvantageous for their spreadibility on the vagina and patient compliance. Hardness and compressibility of formulations prepared with low molecular mass chitosan are low compared to other formulations. Nevertheless, their adhesiveness is comparatively low, which might cause leakage during the use of the formulation and restrict patient compliance. 
Briefly, the compressibility and hardness values of formulations should be low while adhesiveness and cohesiveness values should be high. Also, formulations should show lower numerical values of elasticity, which indicate higher product elasticity. Based on the results of mechanical properties, gels prepared with medium molecular mass chitosan appeared to offer more suitable mechanical properties than gels for vaginal application prepared with low and high molecular mass chitosan.

In addition, the results showed that the addition of active substances in 1 or $2 \%$ concentrations did not significantly affect the adhesiveness, cohesiveness, hardness, compressibility and elasticity of the formulations.

\section{Results of syringeability studies}

The work required to expel samples from a syringe is another important parameter for practical administration of gels with a syringe. This parameter shows the force required for the application of a formulation and affects clinical practice. Previously, Jones et al., reported that increased product viscoelasticity (particularly elasticity) offered increased resistance to product deformation in TPA. In syringeability analysis, this situation causes an increase in work required for compression/expulsion of the formulation (25). Our results on elasticity, hardness and syringeability were in accord with this study and the ranking was: F3 > F2 > F1 (Table II). In addition, significant increases in syringing work were observed as the molecular weight and viscosity of chitosan increased $(p<$ 0.05). F3 required most work to expel the liquid formulation from the syringe (or applicator), which might be disadvantageous for the ease of administering formulations with a syringe or applicator. In addition, the results showed that incorporation of $2 \% \mathrm{MN}$ or $1 \%$ EN into chitosan gels had no significant effect on work required to expel formulations from the syringe.

\section{Results of mucoadhesion studies}

Mucoadhesive properties of formulations play an important role in prolonging the residence time and mucoadhesion should be determined sensitively. A TA-XTplus texture analyzer can be used to measure mucoadhesion of formulations under simulated vaginal conditions, based on the principle of applying tensile strength and shear stress to break the adhesive bond between the test sample and a model membrane (26). Therefore, the mucoadhesive properties of chitosan gels were determined with a TA-XTplus texture analyzer equipped with the A/MUC measuring system.

The results of mucoadhesion studies are presented in Table II. Chitosan is a well-known bioadhesive polysaccharide and its mucoadhesive properties are likely to be based on its cationic character. The mucus layer exhibits anionic structures in sialic acid and sulfonic acid form. Based on ionic interactions between the cationic primary amino groups of chitosan and these anionic substructures of the mucus, mucoadhesion can be achieved. In addition, hydrophobic interactions might contribute to chitosan mucoadhesive properties (4). 
Table II. Results of mechanical properties, work of syringing and work of mucoadhesion of chitosan gels ${ }^{\mathrm{a}}$

\begin{tabular}{|c|c|c|c|c|c|c|c|}
\hline 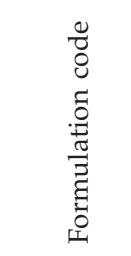 & 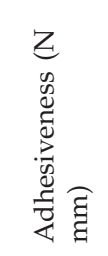 & 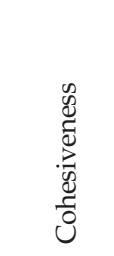 & $\begin{array}{l}\widehat{Z} \\
0 \\
0 \\
\tilde{E} \\
\bar{Z} \\
\bar{Z} \\
\text { I }\end{array}$ & 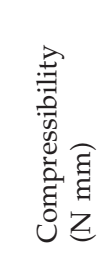 & 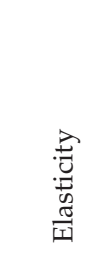 & 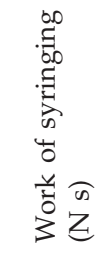 & 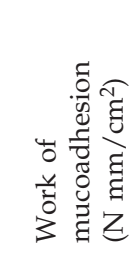 \\
\hline F1 & $\begin{array}{r}0.008 \\
\pm 0.001\end{array}$ & $\begin{array}{r}0.974 \\
\pm 0.023\end{array}$ & $\begin{array}{r}0.009 \\
\pm 0.000\end{array}$ & $\begin{array}{r}0.025 \\
\pm 0.001\end{array}$ & $\begin{array}{r}0.869 \\
\pm 0.062\end{array}$ & $\begin{array}{r}6.893 \\
\pm 0.652\end{array}$ & $\begin{array}{r}222.329 \\
\pm 5.199\end{array}$ \\
\hline F2 & $\begin{array}{r}0.016 \\
\pm 0.001\end{array}$ & $\begin{array}{r}0.912 \\
\pm 0.034\end{array}$ & $\begin{array}{r}0.009 \\
\pm 0.001\end{array}$ & $\begin{array}{r}0.019 \\
\pm 0.002\end{array}$ & $\begin{array}{r}1.005 \\
\pm 0.139\end{array}$ & $\begin{array}{r}21.908 \\
\pm 3.385\end{array}$ & $\begin{array}{r}253.510 \\
\pm 38.041\end{array}$ \\
\hline F3 & $\begin{array}{r}0.062 \\
\pm 0.005\end{array}$ & $\begin{array}{r}1.048 \\
\pm 0.072\end{array}$ & $\begin{array}{r}0.017 \\
\pm 0.002\end{array}$ & $\begin{array}{r}0.032 \\
\pm 0.005\end{array}$ & $\begin{array}{r}1.065 \\
\pm 0.043\end{array}$ & $\begin{array}{r}39.727 \\
\pm 2.177\end{array}$ & $\begin{array}{r}426.538 \\
\pm 63.285\end{array}$ \\
\hline $\mathrm{F} 1+\mathrm{MN}$ & $\begin{array}{r}0.008 \\
\pm 0.001\end{array}$ & $\begin{array}{r}0.925 \\
\pm 0.072\end{array}$ & $\begin{array}{r}0.007 \\
\pm 0.001\end{array}$ & $\begin{array}{r}0.012 \\
\pm 0.002\end{array}$ & $\begin{array}{r}0.985 \\
\pm 0.131\end{array}$ & $\begin{array}{r}7.509 \\
\pm 2.537\end{array}$ & $\begin{array}{r}212.078 \\
\pm 24.193\end{array}$ \\
\hline $\mathrm{F} 2+\mathrm{MN}$ & $\begin{array}{r}0.022 \\
\pm 0.003\end{array}$ & $\begin{array}{r}0.965 \\
\pm 0.031\end{array}$ & $\begin{array}{r}0.010 \\
\pm 0.002\end{array}$ & $\begin{array}{r}0.016 \\
\pm 0.006\end{array}$ & $\begin{array}{r}0.942 \\
\pm 0.099\end{array}$ & $\begin{array}{r}20.055 \\
\pm 0.433\end{array}$ & $\begin{array}{r}252.004 \\
\pm 55.222\end{array}$ \\
\hline $\mathrm{F} 3+\mathrm{MN}$ & $\begin{array}{r}0.075 \\
\pm 0.017\end{array}$ & $\begin{array}{r}1.165 \\
\pm 0.030\end{array}$ & $\begin{array}{r}0.013 \\
\pm 0.001\end{array}$ & $\begin{array}{r}0.016 \\
\pm 0.003\end{array}$ & $\begin{array}{r}1.119 \\
\pm 0.104\end{array}$ & $\begin{array}{r}35.858 \\
\pm 2.857\end{array}$ & $\begin{array}{r}454.886 \\
\pm 22.704\end{array}$ \\
\hline $\mathrm{F} 1+\mathrm{EN}$ & $\begin{array}{r}0.006 \\
\pm 0.000\end{array}$ & $\begin{array}{r}0.990 \\
\pm 0.052\end{array}$ & $\begin{array}{r}0.007 \\
\pm 0.000\end{array}$ & $\begin{array}{r}0.012 \\
\pm 0.001\end{array}$ & $\begin{array}{r}1.004 \\
\pm 0.052\end{array}$ & $\begin{array}{r}6.450 \\
\pm 1.106\end{array}$ & $\begin{array}{r}209.510 \\
\pm 32.027\end{array}$ \\
\hline $\mathrm{F} 2+\mathrm{EN}$ & $\begin{array}{r}0.018 \\
\pm 0.002\end{array}$ & $\begin{array}{r}0.887 \\
\pm 0.010\end{array}$ & $\begin{array}{r}0.011 \\
\pm 0.000\end{array}$ & $\begin{array}{r}0.033 \\
\pm 0.002\end{array}$ & $\begin{array}{r}0.904 \\
\pm 0.076\end{array}$ & $\begin{array}{r}18.377 \\
\pm 2.376\end{array}$ & $\begin{array}{r}277.913 \\
\pm 25.010\end{array}$ \\
\hline $\mathrm{F} 3+\mathrm{EN}$ & $\begin{array}{r}0.120 \\
\pm 0.010 \\
\end{array}$ & $\begin{array}{r}0.978 \\
\pm 0.046 \\
\end{array}$ & $\begin{array}{r}0.036 \\
\pm 0.012 \\
\end{array}$ & $\begin{array}{r}0.036 \\
\pm 0.009 \\
\end{array}$ & $\begin{array}{r}1.154 \\
\pm 0.013 \\
\end{array}$ & $\begin{array}{r}38.620 \\
\pm \quad 6.681 \\
\end{array}$ & $\begin{array}{r}440.472 \\
\pm 36.946 \\
\end{array}$ \\
\hline
\end{tabular}

${ }^{a}$ Mean \pm SD.

Results of mucoadhesion studies were in accord with adhesiveness results obtained from the texture profile analysis of formulations. Significant increases in the work of mucoadhesion were observed as the molecular weight and viscosity of chitosan increased $(p<0.05)$ and F3 showed stronger mucoadhesiveness than other formulations.

\section{Results of release studies}

The in vitro release profiles of $\mathrm{MN}$ and $\mathrm{EN}$ were evaluated in $\mathrm{pH} 4.5$ phosphate buffer at $37{ }^{\circ} \mathrm{C}$ and the results are displayed in Fig. 4. As can be seen, the release of EN and $\mathrm{MN}$ were in range of $50-70 \%$ over 8 hours. In 24 hours, the release rates of $\mathrm{MN}$ from F1, F2 and F3 reached 89, 91 and $96 \%$, respectively. In addition, at the end of 24 hours, the release rates of EN from F1, F2 and F3 were 71, 67, $78 \%$, respectively (data not shown).

Previous studies had shown that drug release might be influenced by gel viscosity. As the viscosity of the gel increased, the release of the drug was expected to be slower. (27). On the other hand, our results showed that the release profiles of MN and EN were not significantly affected by the viscosity of chitosan $(p>0.05)$. When we compared the 

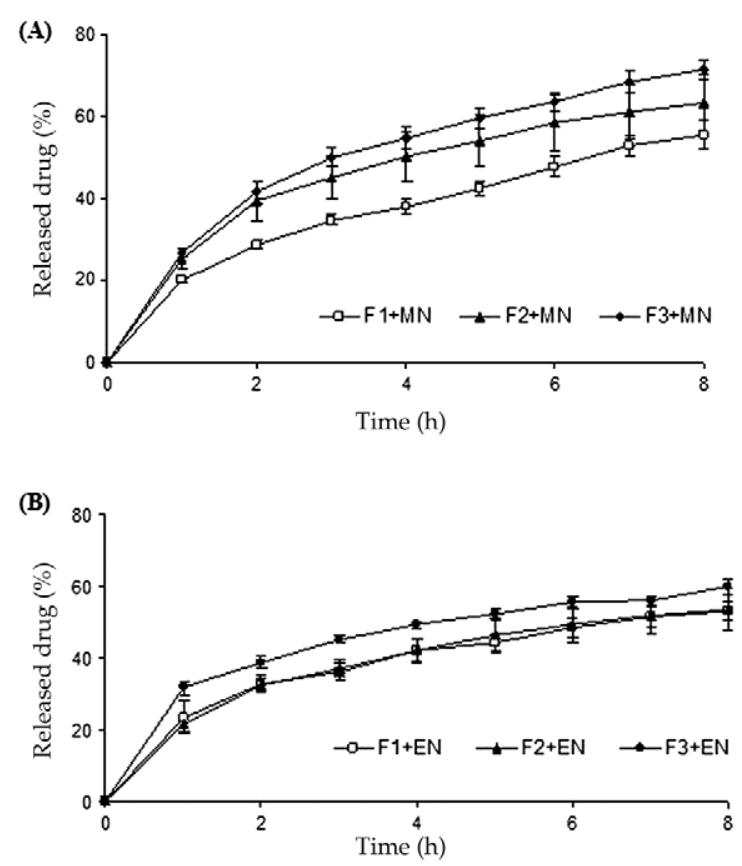

Fig. 4. Dissolution profiles of: a) MN and b) EN from chitosan gels in $\mathrm{pH} 4.5$ phosphate buffer.

release rates of $\mathrm{EN}$ and $\mathrm{MN}$ from the same chitosan formulations, we found that the release rates of MN from F2 and F3 formulations were significantly higher than the release rates of EN from F2 and F3 $(p<0.05)$. On the basis of release studies, it can be concluded that F1, F2 and F3 formulations offer the advantage of controlling both MN and EN release over 8 hours.

\section{Determination of the release mechanism}

An empirical equation (Eq. 3), proposed by Ritger and Peppas, was used for the analysis of release data in gel formulations (16). Application of the general release equation enabled calculation of $n$ and hence identification of the mechanism of release from the formulations. To this aim, the release data were fitted to Eq. 1, and best-fit parameters were calculated. According to this equation, $n=0.5$ indicates case I (Fickian) diffusion or square root of time kinetics, $0.5<n<1.0$ indicates anomalous (non-Fickian) diffusion, $n=1.0$ indicates case II transport, and $n>1.0$ indicates super case II transport for thin film formulations (28).

The values of diffusional exponents $n$, release rate constants $k$ and correlation coefficients $R^{2}$ are listed in Table III. The release exponents $n$ of all formulations ranged from 0.288 to 0.470 , which indicates case I (Fickian) drug release. In this case, diffusion of drug molecules is the controlling mechanism of drug release. 
Z. A. Şenyiğit et al.: Evaluation of chitosan based vaginal bioadhesive gel formulations for antifungal drugs, Acta Pharm. 64 (2014) $139-156$

Table III. Drug release parameters of formulations

\begin{tabular}{cccc}
\hline Formulation code & $\begin{array}{c}\text { Release exponent } \\
(n)\end{array}$ & $\begin{array}{c}\text { Release rate constant } \\
(k)\end{array}$ & $\begin{array}{c}\text { Correlation coefficient } \\
\left(R^{2}\right)\end{array}$ \\
\hline F1 + MN & 0.470 & 1.310 & 0.996 \\
F2 + MN & 0.412 & 1.440 & 0.985 \\
F3 + MN & 0.406 & 1.479 & 0.973 \\
F1 + EN & 0.349 & 1.400 & 0.982 \\
F2 + EN & 0.365 & 1.388 & 0.958 \\
F3 + EN & 0.288 & 1.510 & 0.991 \\
\hline
\end{tabular}

\section{Results of determination of anticandidal activities of formulations}

Chitosan and its derivatives have a spectrum of antimicrobial activity against filamentous fungi, yeasts, Gram-positive and Gram-negative bacteria. Although chitosan is not primarily used as an antimicrobial agent, its utility as an ingredient in pharmaceutical formulations and food has lately gained more interest (29). Therefore, the anticandidal activity of chitosan gels against the standard Candida albicans ATCC 90028 strain was evaluated and the results are given in Table IV.

Although chitosan was known to have anticandidal action against Candida albicans, our results showed that F1, F2 and F3 formulations without MN or EN did not exhibit any antimicrobial activity. Chitosan's in vitro antimicrobial activity is influenced by various intrinsic and extrinsic factors, such as chitosan itself (type, molecular mass, deacetylation degree, solvent and concentration) and the environmental conditions (test strain, its physiological state and the bacterial culture medium, $\mathrm{pH}$, temperature, ionic strength, metal ions, EDTA, organic matter) (30). Therefore, we might conclude that our formulations did not show antimicrobial activity due to some of these intrinsic or extrinsic parameters. It was stated in recent years that the antimicrobial properties of chitosan might have a negative impact on healthy vaginal microflora and that its vaginal use for treat-

Table VI. Inhibition zone diameters of formulations

\begin{tabular}{cc}
\hline Formulation & Diameter of inhibition zone $(\mathrm{mm})$ \\
\hline F1 & 0 \\
F2 & 0 \\
F3 & 0 \\
F1 + MN & $33.6 \pm 1.5$ \\
F2 + MN & $26.3 \pm 0.5$ \\
F3 + MN & $28.3 \pm 0.5$ \\
F1 + EN & $31 \pm 1$ \\
F2 + EN & $30.3 \pm 1.5$ \\
F3 + EN & $30.0 \pm 2$ \\
\hline
\end{tabular}


ment of chronic diseases requires caution (4). From this point of view, we might say that the absence of antimicrobial effect in our formulations without active substances might be an advantageous outcome.

Chitosan gels with active substances showed anticandidal activity with inhibition diameters between $26-34 \mathrm{~mm}$. There were no significant differences in this anticandidal activity among the formulations and active substances.

\section{Results of in vivo distribution studies}

Vaginal retention of gels is an important parameter to be evaluated in order to achieve efficacy. Optimally, a mucoadhesive vaginal gel must be distributed over the mucosa, maintaining an epithelial coating layer (31). To date, several in vitro and in vivo methods have been developed and used to evaluate the distribution and retention of formulations in the vagina $(31,32)$. Imaging studies such as magnetic resonance imaging and gamma scintigraphy still remain the most reliable methods for in vivo assessment of vag-

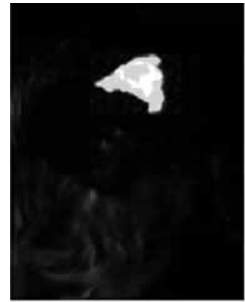

F1 (At the beginning)

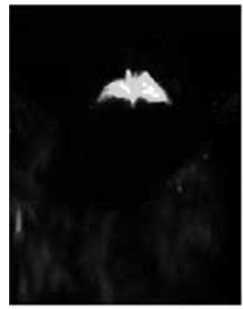

F2 (At the beginning)

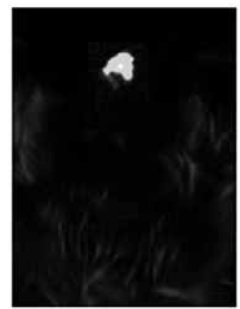

F3 (At the beginning)

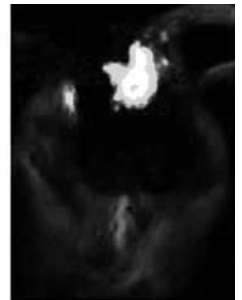

F1 (24 hours later)

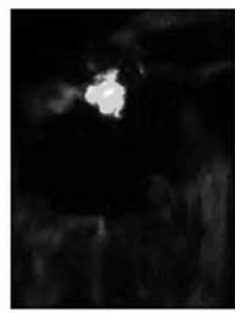

F2 (24 hours later)

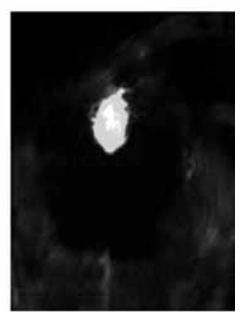

F3 (24 hours later)

Fig. 5. Vaginal retention of formulations. 
inal distribution and retention (33). In our study, we evaluated vaginal retention using a new imaging method: Odyssey Infrared Imaging System with MousePOD ${ }^{\mathrm{TM}}$ adapter (LI-COR Biosciences). The benefit of this method is that live animals are used and that they are not exposed to radiation like in radio-imaging studies (34). Near-infrared images of rats administered RDye 800RS Carboxylate dye (peak absorption at $786 \mathrm{~nm}$ ) loaded chitosan gels are shown in Fig. 5 .

The in vivo distribution studies showed that F1, F2 and F3 formulations remained in the vaginal mucosa $24 \mathrm{~h}$ after application. It was stated in previous studies that the rheology of vaginal gel could be considered as one of the most important factors affecting gel distribution and retention time in the vagina (35). Selection of correct viscosity of a gel can be decisive for its adequate retention and distribution in the vagina. On the other hand, our results showed that F1, F2 and F3 formulations with different chitosan viscosity values remained on the vaginal mucosa up to 24 hours and no significant difference was found among the formulations.

Previously, Di Fabio et al. evaluated the potential effectiveness of microbicides on vaginal HIV-1 transmission formulated with two different polymers (carbopol and hydroxyethylcellulose). The results showed that it was essential to achieve a uniform distribution on vaginal mucosa in order to form an effective barrier. However, the gel with a high viscosity value was difficult to apply and distribute on the vagina of mice. Then, polymers with lower viscosity were examined to achieve a uniform distribution over the entire vagina/cervix and the results showed that vaginal transmission of HIV was successfully prevented by the application of this formulation (36). On the other hand, gels with low viscosity values may result in leakage of the administered formulation from the vagina and restrict the efficacy of the formulation or patient compliance. Therefore, although no significant difference in vaginal retention time was noticed between the formulations, F2 seems to be more suitable than F1 and F3 considering the mechanical, mucoadhesive and syringeability properties. Anyway, in further in vivo studies, the difference between the formulations should be evaluated in more detail.

\section{CONCLUSIONS}

The aim of this study was the evaluation of chitosan (different molecular mass and viscosity values) for the preparation of mucoadhesive vaginal gels. For this purpose, miconazole nitrate and econazole nitrate were chosen as imidazole derivative active substances. The gels were first comprehensively evaluated in in vitro characterization studies (rheological evaluation, texture profile analysis, mucoadhesion, syringeability and release studies). In addition, vaginal retention of the gels was investigated in vivo through a distribution study on rats. Based on the results obtained from these studies, it can be concluded that vaginal gels prepared with medium molecular mass chitosan might be effectively used for different antifungal agents for the treatment of vaginal candidiasis, since they have high mucoadhesiveness, suitable mechanical and release properties with good retention in the vagina.

Acknowledgements. - This work is a part of the authors' research project (No. 106/S/196) supported by the Scientific and Technological Research Council of Turkey (TUBITAK). 


\section{REFERENCES}

1. B. Albertini, N. Passerini, M. Di Sabatino, B. Vitali, P. Brigidi and L. Rodriguez, Polymer-lipid based mucoadhesive microspheres prepared by spray-congealing for the vaginal delivery of econazole nitrate, Eur. J. Pharm. Sci. 36 (2009) 591-601; DOI: 10.1016/j.ejps.2008.12.009.

2. J. A. Kamarashev and S. G. Vassileva, Dermatologic diseases of the vulva, Clin. Dermatol. 15 (1997) 53-65; DOI: 10.1016/j.bbr.2011.03.031.

3. R. Gurny, J. M. Meyer and N. A. Peppas, Bioadhesive intraoral release systems: design, testing and analysis, Biomaterials 5 (1984) 336-340; DOI: 10.1016/0142-9612(84)90031-0.

4. A. Bernkop-Schnürch and S. Dünnhaupt, Chitosan-based drug delivery systems, Eur. J. Pharm. Biopharm. 81 (2012) 463-469; DOI: 10.1016/j.ejpb.2012.04.007.

5. C. Valenta, The use of mucoadhesive polymers in vaginal delivery, Adv. Drug Deliv. Rev. 57 (2005) 1692-1712; DOI: 10.1016/j.addr.2005.07.004.

6. J. E. Codd and P. B. Deasy, Formulation development and in vivo evaluation of a novel bioadhesive lozenge containing a synergistic combination of antifungal agents, Int. J. Pharm. 173 (1998) 13-24; DOI: 10.1016/S0378-5173(98)00228-2.

7. D. S. Jones, A. D. Woolfson and A. F. Brown, Textural, viscoelastic and mucoadhesive properties of pharmaceutical gels composed of cellulose polymers, Int. J. Pharm. 151 (1997) 223-233; DOI: 10.1016/S0378-5173(97)04904-1.

8. S. K. Yellanki, N. K. Nerella, S. Goranti and S. K. Deb, Development of metronidazole intravaginal gel for the treatment of bacterial vaginosis: Effect of mucoadhesive natural polymers on the release of metronidazole, Int. J. Pharm. Tech. Res. 2 (2010) 1746-1750.

9. D. S. Jones, A. D. Woolfson and A. F. Brown, Textural analysis and flow rheometry of novel, bioadhesive antimicrobial oral gels, Pharm. Res. 14 (1997) 450-457; DOI: 10.1023/A: 1012091231023.

10. G. P. Andrews, S. P. Gorman and D. S. Jones, Rheological characterisation of primary and binary interactive bioadhesive gels composed of cellulose derivatives designed as ophthalmic viscosurgical devices, Biomaterials 26 (2005) 571-580; DOI: 10.1016/j.biomaterials.2004.02.062.

11. D. S. Jones, A. D. Woolfson and J. Djokic, Texture profile analysis of bioadhesive polymeric semisolids: mechanical characterization and investigation of interactions between formulation components, J. Appl. Polym. Sci. 61 (1996) 2229-2234; DOI: 10.1002/(SICI)1097-4628(19960919)61.

12. D. S. Jones, A. D. Woolfson, A. F. Brown, W. A. Coulter, C. McClelland and C. R. Irwin, Design, characterisation and preliminary clinical evaluation of a novel mucoadhesive topical formulation containing tetracycline for the treatment of periodontal disease, J. Control. Release 67 (2000) 357-368; DOI: 10.1016/S0168-3659(00)00231-5.

13. G. Sandri, M. C. Bonferoni, S. Rossi, F. Ferrari, M. Mori, C. Del Fante, C. Perotti, L. Scudeller and C. Caramella, Platelet lysate formulations based on mucoadhesive polymers for the treatment of corneal lesions, J. Pharm. Pharmacol. 63 (2011) 189-198; DOI: 10.1111/j.2042-7158.2010. 01208.x.

14. C. E. Kast, C. Valenta, M. Leopold and A. Bernkop-Schnürch, Design and in vitro evaluation of a novel bioadhesive vaginal drug delivery system for clotrimazole, J. Control. Release 81 (2002) 347-354; DOI: 10.1016/S0168-3659(02)00077-9.

15. N. A. Peppas, Analysis of fickian and non-fickian drug release polymers, Pharm. Acta Helv. 60 (1985) 110-111.

16. P. L. Ritger and N. A. Peppas, A simple equation for description of solute release II. Fickian and anomalous release from swellable devices, J. Control. Release 5 (1987) 37-42; DOI: 10.1016/ 0168-3659(87)90035-6.

17. J. Parekh and S. Chanda, In vitro antimicrobial activity and phytochemical analysis of some Indian medicinal plants, Turk. J. Biol. 31 (2007) 53-58. 
18. X. He, J. Ma, A. E. Mercado, W. Xu and E. Jabbari, Cytotoxicity of paclitaxel in biodegradable self-assembled core-shell poly (lactide-co-glycolide ethylene oxide fumarate) nanoparticles, Pharm. Res. 25 (2008) 1552-1562; DOI: 10.1007/s11095-007-9513-z.

19. K. Vermani and S. Garg, The scope and potential of vaginal drug delivery, PSTT 3 (2000) 359-364; DOI: 10.1016/S1461-5347(00)00296-0.

20. S. Uppuluri, S. E. Keinath, D. A. Tomalia and P. R. Dvornic, Rheology of dendrimers. I. Newtonian flow behavior of medium and highly concentrated solutions of polyamidoamine (PAMAM) dendrimers in ethylenediamine (EDA) solvent, Macromolecules 31 (1998) 4498-4510; DOI: 10.1021/ ma971199b.

21. H. Qi, W. Chen, C. Huang, I. Li, C. Chen, W. Li and C. Wu, Development of a poloxamer analogs/carbopol-based in situ gelling and mucoadhesive ophthalmic delivery system for puerarin, Int. J. Pharm. 337 (2007) 178-187; DOI: 10.1016/j.ijpharm.2006.12.038.

22. G. Dumortier, J. L. Grossiord, M. Zuber, G. Couarraze and J. C. Chaumeil, Rheological study of a thermoreversible morphine gel, Drug Dev. Ind. Pharm. 17 (1991) 1255-1265; DOI: 10.3109/ 03639049109043858.

23. K. Dillen, W. Weyenberg, J. Vandervoort and A. Ludwig, The influence of the use of viscosifiying agents as dispersion media on the drug release properties from PLGA nanoparticles, Eur. J. Pharm. Biopharm. 58 (2004) 539-549; DOI: 10.1016/j.ejpb.2004.03.035.

24. W. Argüelles-Monal, F. M. Goycoolea, C. Peniche and I. Higuera-Ciapara, Rheological study of the chitosan/glutaraldehyde chemical gel system, Polym. Gels Netw. 6 (1998) 429-440; DOI: 10.1016/S0966-7822(98)00032-X.

25. D. S. Jones, A. D. Woolfson and A. F. Brown, Design, characterisation and preliminary clinical evaluation of a novel mucoadhesive topical formulation containing tetracycline for the treatment of periodontal disease, J. Control. Release 67 (2000) 357-368; DOI: 10.1016/S0168-3659(00) 00231-5.

26. E. Baloglu, Z. Ay Senyigit, S. Y. Karavana and A. Bernkop-Schnürch, Strategies to prolong the intravaginal residence time of drug delivery systems, Pharm. Pharmac. Sci. 12 (2009) 312-336.

27. S. Y. Karavana, Z. Ay Senyigit, S. Hilmioğlu-Polat, D. Y. Metin, O. Zekioğlu and E. Baloğlu, Mucoadhesive In situ gel formulations of miconazole nitrate for the treatment of mucosal candidiasis, Lat. Am. J. Pharm. 31 (2012) 821-829.

28. J. Siepmann and N. A. Peppas, Modeling of drug release from delivery systems based on hydroxypropyl methylcellulose (HPMC), Adv. Drug Deliv. Rev. 48 (2001) 139-157; DOI: 10.1016/ S0169-409X(01)00112-0.

29. A. A. Tayela, S. Moussa, W. F. El-Tras, D. Knittel, K. Opwis and E. Schollmeyer, Anticandidal action of fungal chitosan against Candida albicans, Int. J. Biol. Macromol. 47 (2010) 454-457; DOI: 10.1016/j.ijbiomac.2010.06.011.

30. R. Muzzarelli, R. Tarsi, O. Filippini, E. Giovanetti, G. Biagini and P. E. Varaldo, Antimicrobial properties of N-carboxybutyl chitosan, Antimicrob. Agents Chemother. 34 (1990) 2019-2023; DOI: 10.1128/AAC.34.10.2019.

31. A. R. Geonnotti, J. J. Peters and D. F. Katz, Erosion of microbicide formulation coating layers: effects of contact and shearing with vaginal fluid or semen, J. Pharm. Sci. 94 (2005) 1705-1712; DOI: $10.1002 /$ jps.20386.

32. K. Vermani, S. Garg and L. J. Zaneveld, Assemblies for in vitro measurement of bioadhesive strength and retention characteristics in simulated vaginal environment, Drug Dev. Ind. Pharm. 28 (2002) 1133-1146; DOI: 10.1081/DDC-120014580.

33. B. E. Chatterton, S. Penglis, J. C. Kovacs, B. Presnell and B. Hunt, Retention and distribution of two 99mTc-DTPA labelled vaginal dosage forms, Int. J. Pharm. 271 (2004) 137-143; DOI: 10.1016/ j.ijpharm.2003.11.006. 
34. S. Y. Karavana, E. H. Gökçe, S. Rençber, S. Özbal, Ç. Pekçetin, P. Güneri and G. Ertan, A new approach to the treatment of recurrent aphthous stomatitis with bioadhesive gels containing cyclosporine A solid lipid nanoparticles: in vivo/in vitro examinations, Int. J. Nanomed. 7 (2012) 5693-5704; DOI: 10.2147 /IJN.S36883.

35. D. H. Owen, J. J. Peters and D. F. Katz, Rheological properties of contraceptive gels, Contraception 62 (2000) 321-326; DOI: 10.1016/S0010-7824(00)00184-0.

36. S. Di Fabio, J. Van Roey, G. Giannini, G. van den Mooter, M. Spada, A. Binelli, M. F. Pirillo, E. Germinario, F. Belardelli, M. P. de Bethune and S. Vella, Inhibition of vaginal transmission of HIV-1 in hu-SCID mice by the non-nucleoside reverse transcriptase inhibitor TMC120 in a gel formulation, AIDS 17 (2003) 1597-1604; DOI: 10.1097/00002030-200307250-00003. 